\title{
Expansions in Series of Spherical Harmonics.
}

\author{
By Dr T. M. MacRobert.
}

(Read and Received 7th December 1923.)

\$1. A Generalisation of Christoffel's Summation Formula. The Recurrence Formula

$$
(n-m+1) P_{n+1}^{m}(z)-(2 n+1) z P_{n}^{m}(z)+(n+m) P_{n-1}^{m}(z)=0
$$

is valid for all values of $n$ and $m$; but, if $m$ is a positive integer, $P_{m-1}^{m}(z)=0$, hence, when $n=m$, (1) becomes

$$
P_{m+1}^{m}(z)-(2 m+1) z P_{m}^{m}(z)=0 .
$$

In (1) write $x$ and $y$ for $z$ in succession, multiply the resulting equations by $P_{n}^{m}(y)$ and $P_{n}^{m}(x)$ respectively, substract, and obtain the formula

$$
\begin{aligned}
& (2 n+1)(x-y) P_{n}^{n}(x) P_{n}^{n}(y) \\
& =(n-m+1)\left\{P_{n+1}^{n}(x) P_{n}^{m}(y)-P_{n+1}^{m}(y) P_{n}^{m}(x)\right\} \\
& \quad-(n+m)\left\{P_{n}^{m}(x) P_{n-1}^{n}(y)-P_{n}^{m}(y) P_{n-1}^{m}(x)\right\} .
\end{aligned}
$$

Similarly from (2) it can be deduced that, when $m$ is a positive integer,

$$
(2 m+1)(x-y) P_{m}^{m}(x) P_{m}^{m}(y)=\left\{P_{m+1}^{m}(x) P_{m}^{m}(y)-P_{m+1}^{m}(y) P_{m}^{m}(x)\right\}
$$

In (3) put $n=m+r$, multiply by $r$ !, and divide by

$$
(2 m+1)(2 m+2) \ldots(2 m+r)
$$

this gives

$$
\begin{aligned}
& \frac{r !(2 m+2 r+1)}{(2 m+1)(2 m+2) \ldots(2 m+r)}(x-y) P_{m+r}^{m}(x) P_{m+r}^{m}(y) \\
= & \frac{(r+1) !}{(2 m+1)(2 m+2) \ldots(2 m+r)}\left\{P_{m+r+1}^{m}(x) P_{m+r}^{m}(y)-P_{m+r+1}^{m}(y) P_{m+r}^{m}(x)\right\} \\
- & \frac{r !}{(2 m+1)(2 m+2) \ldots(2 m+r-1)}\left\{P_{m+r}^{m}(x) P_{m+r-1}^{m}(y)-P_{m+r}^{m}(y) P_{m+r-1}^{m}(x)\right\} .
\end{aligned}
$$


Now in (5) put $r=1,2, \ldots n$ in succession, and add the resulting equations to $(4)$; then, if

$$
\begin{aligned}
& \Omega_{n+1}=(2 m+1) P_{m}^{m}(x) P_{m}^{m}(y)+\frac{1 !(2 m+3)}{(2 m+1)} P_{m+1}^{m}(x) P_{m+1}^{m}(y) \\
&+\frac{2 !(2 m+5)}{(2 m+1)(2 m+2)} P_{m+2}^{m}(x) P_{m+2}^{m}(y)+\ldots \ldots \\
&+\frac{n !(2 m+2 n+1)}{(2 m+1)(2 m+2) \ldots(2 m+n)} P_{m+n}^{m}(x) P_{m+n}^{m}(y), \ldots \ldots(6) \\
&(x-y) \Omega_{n+1}=\frac{(n+1) !}{(2 m+1)(2 m+2) \ldots(2 m+n)} \\
&\left\{P_{m+n+1}^{m}(x) P_{m+n}^{m}(y)-P_{m+n+1}^{m}(y) P_{m+n}^{m}(x)\right\} \ldots(7)
\end{aligned}
$$

\$2. Expansion of a Function in a Series of Associated Legendre Functions. If it is assumed that the expansion

$$
f(x)=\sum_{r=0}^{\infty} A_{r} P_{m+r}^{m}(x),
$$

where $m$ is a positive integer, is valid for $-1 \leqq x \leqq 1$, the coefficient $A_{r}$ may be evaluated by multiplying the equation by $P_{m+r}^{m}(x)$ and integrating from $x=-1$ to $x=+1$; thus

$$
\begin{gathered}
\int_{-1}^{1} f(y) P_{m+r}^{m}(y) d y=A_{r} \int_{-1}^{1}\left\{P_{m+r}^{m}(y)\right\}^{2} d y \\
=A_{r}(-1)^{m} \frac{2}{2 m+2 r+1} \frac{(2 m+r) !}{r !} .
\end{gathered}
$$

Hence, if $\Sigma_{n+1}$ denotes the first $n+1$ terms of (8),

$$
\begin{aligned}
& \Sigma_{n+1}=\frac{(-1)^{m}}{2 \cdot(2 m) !} \int_{-1}^{1} f(y) \Omega_{n+1} d y \\
& =\frac{(-1)^{m}(n+1) !}{2 \cdot(2 m+n) !} \int_{-1}^{1} f(y) \frac{P_{m+n+1}^{m}(x) P_{m+n}^{m}(y)-P_{m+n+1}^{m}(y) P_{m+n}^{m}(x)}{x-y} d y,
\end{aligned}
$$

by (6) and (7).

Now in this formula write $x=\cos \theta, y=\cos \phi$, and replace the Associated Legendre Functions by their Asymptotic Expansions; these are given by the equation

$$
\begin{aligned}
& P_{n}^{m}(\cos \theta)=e^{-i m \pi i} \frac{e^{i \pi / 4}}{\sqrt{ }(2 \pi i \sin \theta)} \frac{\Pi(n+m)}{\Pi\left(n+\frac{1}{2}\right)} \\
& \times\left[\begin{array}{l}
\left.e^{-\frac{1}{2 m \pi i+2 \pi i-(n+1) \theta i} F\left(\frac{1}{2}-m, \frac{1}{2}+m, n+\frac{3}{2},\right.}-\frac{e^{-i \theta}}{2 i \sin \theta}\right) \\
+e^{\frac{1 m \pi i-t \pi i+(n+1) \theta i}{2}} F\left(\frac{1}{2}-m, \frac{1}{2}+m, n+\frac{3}{2}, \frac{e^{i \theta}}{2 i \sin \theta}\right)
\end{array}\right]
\end{aligned}
$$

7 Vol. 42 
Then from (9) and (10)

$$
\begin{aligned}
& \Sigma_{n+1}=\frac{(n+1) !}{(2 m+n) !} \frac{\Pi(2 m+n+1) \Pi(2 m+n)}{\pi \cdot \Pi\left(m+n+\frac{3}{2}\right) \Pi\left(m+n+\frac{1}{2}\right)}\left(1+k^{\prime}\right) \\
& \times \int_{0}^{\pi} \frac{f(\cos \phi)}{\sqrt{(\sin \theta \sin \phi)}} \\
& \left\{\begin{array}{l}
\cos \left\{\left(m+n+\frac{3}{2}\right) \theta-\frac{1}{4} \pi+\frac{1}{2} m \pi\right\} \cos \left\{\left(m+n+\frac{1}{2}\right) \phi-\frac{1}{4} \pi+\frac{1}{2} m \pi\right\} \\
-\cos \left\{\left(m+n+\frac{3}{2}\right) \phi-\frac{1}{4} \pi+\frac{1}{2} m \pi\right\} \cos \left\{\left(m+n+\frac{1}{2}\right) \theta-\frac{1}{4} \pi+\frac{1}{2} m \pi\right\}
\end{array}\right\} \frac{\sin \phi d \phi}{(\cos \theta-\cos \phi)},
\end{aligned}
$$

where $k^{\prime} \rightarrow 0$ as $n \rightarrow \infty$. Again, since $\Pi(n+\alpha) \sim \Pi(n) \times n^{a}$

$$
\begin{aligned}
& \Sigma_{n+1}=\frac{1}{\pi}(1+k)
\end{aligned}
$$

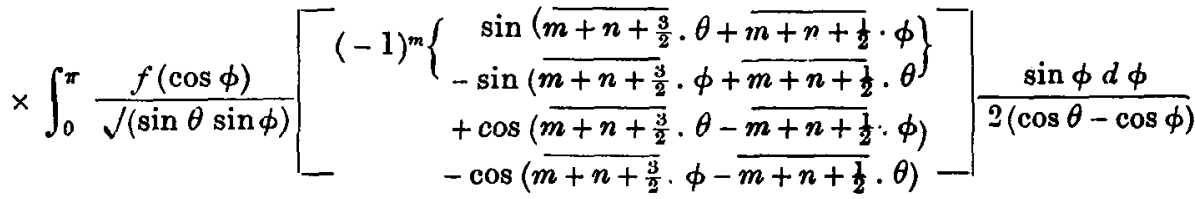$$
=\frac{1}{\pi}(1+k) \int_{0}^{\pi} \frac{f(\cos \phi)}{\sqrt{ }(\sin \theta \sin \phi)}\left[\begin{array}{c}
(-1)^{m}\left\{\begin{array}{c}
\left.\sin \lambda \cos \frac{1}{2}(\theta-\phi)+\cos \lambda \sin \frac{1}{2}(\theta-\phi)\right\}- \\
-\sin \lambda \cos \frac{1}{2}(\theta-\phi)+\cos \lambda \sin \frac{1}{2}(\theta-\phi)
\end{array}\right\} \\
+\cos \mu \cos \frac{1}{2}(\theta+\phi)-\sin \mu \sin \frac{1}{2}(\theta+\phi) \\
-\cos \mu \cos \frac{1}{2}(\theta+\phi)-\sin \mu \sin \frac{1}{2}(\theta+\phi)
\end{array}\right]
$$

$$
\frac{\sin \phi d \phi}{2(\cos \theta-\cos \phi)}
$$

where $\lambda=(m+n+1)(\theta+\phi), \mu=(m+n+1)(\theta-\phi)$, and $k \rightarrow 0$ as $n \rightarrow \infty$

$$
=\frac{1}{\pi}\langle 1+k) \int_{0}^{\pi} \frac{f(\cos \phi)}{\sqrt{ }(\sin \theta} \frac{1}{\sin \phi)}\left[\begin{array}{l}
\sin \{(m+n+1)(\theta-\phi)\} \sin \frac{1}{2}(\theta+\phi) \\
-(-1)^{m} \cos \{(m+n+1)(\theta+\phi)\} \sin \frac{1}{2}(\theta-\phi)
\end{array}\right]
$$

$$
\frac{\sin \phi d \phi}{2 \sin \frac{1}{2}(\theta-\phi) \sin \frac{1}{2}} \overline{(\theta+\phi)} \text {. }
$$

When $n \rightarrow \infty$, this expression, since the integral is the sum of two Dirichlet Integrals, tends to the value

$$
\frac{1}{2}\{f(\cos \overline{\theta+0})+f(\cos \overline{\theta-0})\} \text { or } \frac{1}{2}\{f(x+0)+f(x-0)\},
$$

subject to the usual restrictions on the function $f(x)$, and provided that $-1<x<1$.

As, however, the asymptotic expansion does not hold when $\phi=0$ or $\pi$, it is necessary to show that the integrand in (9) remains finite in the neighbourhood of the limits of integration. This can be proved as follows. 
From the contour integral expression for the hypergeometric function we have, if $J$ denotes the expression

$$
\begin{aligned}
\left(1+e^{2 \pi i m}\right)\left(1-e^{2 \pi i(n-m)}\right) B\left(m+\frac{1}{2}, n-m+1\right) F\left(\frac{1}{2}-m, \frac{1}{2}+m, n+\frac{3}{2}, z\right), \\
J=\int^{(1+, 0+, 1-, 0-)} \zeta^{m-1}(1-\zeta)^{n-m}(1-z \zeta)^{m-\frac{1}{2}} d \zeta \\
=\int^{(1+, 0+, 1-, 0-)} \zeta^{2 m-1}(1-\zeta)^{n-m}\left(1-\frac{1}{z \zeta}\right)^{m-1} d \zeta(-z)^{m-\frac{1}{2}} ;
\end{aligned}
$$

here expand $\left(1-\frac{1}{z \zeta}\right)^{m-t}$ in the form

$$
\begin{aligned}
1 & +\frac{\frac{1}{2}-m}{1 !(z \zeta)}+\frac{\left(\frac{1}{2}-m\right)\left(\frac{3}{2}-m\right)}{2 !(z \zeta)^{2}}+\ldots+\frac{\left(\frac{1}{2}-m\right)\left(\frac{3}{2}-m\right) \cdot\left(-\frac{3}{2}\right)}{(m-1) !(z \zeta)^{m-1}} \\
& +\frac{\left(\frac{1}{2}-m\right)\left(\frac{3}{2}-m\right) . .\left(-\frac{1}{2}\right)}{m !(z \zeta)^{m}} \int_{0}^{1} m(1-t)^{m-1}\left(1-\frac{t}{z \zeta}\right)^{-\frac{1}{2}} d t
\end{aligned}
$$

and carry out the integration of the first $m$ terms. If $m$ is a positive integer (we assume for the time being that $n$ is not an integer), these $m$ integrals will all contain a factor $\left(1-e^{4 \pi i m}\right)$, which, of course, has the value zero. Hence

$$
\begin{aligned}
J & =\frac{\left(\frac{1}{2}-m\right)\left(\frac{3}{2}-m\right) \ldots\left(-\frac{1}{2}\right)}{m !}(-1)^{m} \int_{0}^{1} m(1-t)^{m-1} t-\frac{1}{\zeta} d t \\
& \times \int^{(1+, 0+, 1-, 0-)} \zeta^{m-\frac{1}{2}}(1-\zeta)^{n-m}\left(1-\frac{z \zeta}{t}\right)^{-\frac{1}{t}} d \zeta \\
& =\frac{\Gamma\left(m+\frac{1}{2}\right)}{\Gamma\left(\frac{1}{2}\right) m !}\left(1+e^{2 \pi i m}\right)\left(1-e^{2 \pi i(n-m)}\right) \\
& \times B\left(m+\frac{1}{2}, n-m+1\right) \int_{0}^{1} m(1-t)^{m-1} t-\frac{1}{2} F\left(\frac{1}{2}, \frac{1}{2}+m, n+\frac{3}{2}, \frac{z}{t}\right) d t .
\end{aligned}
$$

Therefore, if $m$ is a positive integer,

where

$$
F\left(\frac{1}{2}-m, \frac{1}{2}+m, n+\frac{3}{2}, z\right)=\frac{1}{B\left(m, \frac{1}{2}\right) B\left(m+\frac{1}{2}, n-m+1\right)} I,
$$

$$
I=\int_{0}^{1}(1-t)^{m-1} t^{-t} d t \int_{0}^{1} \xi^{m-\frac{1}{t}}(1-\zeta)^{n-m}\left(1-\frac{z \zeta}{t}\right)^{-\frac{1}{1}} d \zeta .
$$

Now, if $z=\frac{1}{2} \pm \frac{1}{2} i \cot \theta$,

$$
\begin{aligned}
& \left|1-\frac{z \zeta}{t}\right|=\sqrt{ }\left\{\left(1-\frac{\zeta}{2 t}\right)^{2}+\left(\frac{\zeta \cot \theta}{2 t}\right)^{2}\right\}=\sqrt{\left\{1-\frac{\zeta}{t}+\frac{\zeta^{2} \operatorname{cosec}^{2} \theta}{4 t^{2}}\right\}}
\end{aligned}
$$

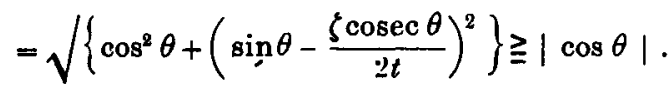


Hence $\left|\left(1-\frac{z \zeta}{t}\right)^{-t}\right| \leqq \sqrt{ }(|\sec \theta|)$,

so that

$$
\left|F\left(\frac{1}{2}-m, \frac{1}{2}+m, n+\frac{3}{2}, \frac{1}{2} \pm \frac{1}{2} i \cot \theta\right)\right| \leqq \sqrt{ }(|\sec \theta|) .
$$

Now the arguments of the hypergeometric functions in (10) are of the forms $\frac{1}{2} \pm \frac{1}{2} i \cot \theta$, where $0<\theta<\pi$ : therefore since, when $n$ is an integer, $P_{n}^{m}(\cos \phi) \sqrt{ }(2 \pi \sin \phi)$ is continuous at $\phi=0$ and $\phi=\pi$,

$$
\left|P_{n}^{m}(\cos \phi) \sqrt{ }(2 \pi \sin \phi)\right| \leqq \frac{\Pi(n+m)}{\Pi\left(n+\frac{1}{2}\right)} 2 \sqrt{ }(\sec \epsilon)
$$

for $0 \leqq \phi \leqq \epsilon$ and $\pi-\epsilon \leqq \phi \leqq \pi$.

If now $\epsilon$ be chosen so small that $\epsilon<\theta<\pi-\epsilon$, it follows that the expression

$$
\frac{(n+1) !}{(2 m+n) !} \frac{P_{m+n+1}^{m}(\cos \theta) P_{m+n}^{m}(\cos \phi)-P_{m+n+1}^{m}(\cos \phi) P_{m+n}^{m}(\cos \theta)}{\cos \theta-\cos \phi} \sin \phi
$$

remains finite for $0 \leqq \phi \leqq \epsilon$ and for $\pi-\epsilon \leqq \phi \leqq \pi$; thus the integrals between these limits can be made arbitrarily small by decreasing $\epsilon$, and the remaining part of (11) then gives the required result.

Note. - By combining this theorem with Fourier's Expansion it can be shown that a continuous function $f(\theta, \phi)$ of $\theta$ and $\phi$ can be expanded in a doubly-infinite series of terms of the types $\cos m \phi P_{n}^{m}(\cos \theta), \sin m \phi P_{n}^{m}(\cos \theta)$, where $m$ and $n$ are positive integers. 